\title{
Synthesis, Characterization and Electrochemical Property of Polypyrrole Nanoparticles $†$
}

\author{
S. MUNUSAMY ${ }^{\mathrm{a}}, \mathrm{K}$. GIRIBABU ${ }^{\mathrm{a}}$, R. MANIGANDAN ${ }^{\mathrm{a}}$, \\ S. MUTHAMIZH ${ }^{\mathrm{a}}$, L. VIJAYALAKSHMI ${ }^{\mathrm{b}}$ and V. NARAYANAN ${ }^{\mathrm{a} *}$ \\ ${ }^{a}$ Department of Inorganic Chemistry, University of Madras, Guindy Campus, \\ Chennai-600025, India

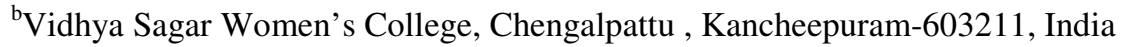 \\ vnnara@yahoo.co.in
}

Received 19 January 2013 / Accepted 5 February 2013

\begin{abstract}
Polypyrrole nanoparticles were synthesized by chemical polymerization method using ammonium persulphate as an oxidant. The synthesized polypyrrole nanoparticles were characterized by FT-IR, UV-Visible, FESEM and CV. The structure of polypyrrole nanoparticles were confirmed by FT-IR spectroscopy. The UV-Vis spectra of polypyrrole nanoparticles show $\pi-\pi^{*}$ and $n-\pi^{*}$ transitions. The morphological properties were characterized by FESEM and it shows that the synthesized polymer has agglomerated morphology. The electrochemical properties of the polypyrrole nanoparticles were studied by cyclic voltammetric method. It is reasonable to expect that the synthesized polypyrrole nanoparticles will exhibit promising applications in the field of electrochemical sensor.
\end{abstract}

Keyword: Polypyrrole, Nanoparticles, FT-IR spectroscopy, Cyclic voltammetry

\section{Introduction}

The interest in conducting polymers began in the 1970's, when it was found that the electrical conductivity of polyacetylene, a semi-conductor when pristine, could be increased by over fifteen orders of magnitude by treatment with oxidizing agents such as iodine. In heterocyclic polymers, where two non-degenerate regions are separated by a topological defect, the formation of single solutions like polyacetylene is energetically unfavorable $^{1}$ and paired sites are formed ${ }^{2}$. This is the case for polythiophene, polyindole or polypyrrole, which can be drawn with either aromatic or quinoid structures, of which the latter possesses a higher energy configuration. Two neutral radicals on a single chain will recombine to eliminate the structural defect ${ }^{3-5}$, although, if one is charged, a polaron is formed, which is delocalized over about four rings. However, when both defects are charged, they are predicted to pair up to form a 'bipolaron' ${ }^{6}$, consisting of a doubly charged defect with no spin and extending over a number of rings to a similar polaron. At higher doping levels, bipolarons may also combine to form 'bipolaron bands' within the gap. Among the

$\dagger$ Presented to the National Conference on Chemistry Solutions at SRM University, India 
conducting polymers known to date, the ones based upon polypyrrole have attracted special interest because of their high conductivity, their ease and high flexibility in preparation, their stability and good mechanical properties.

Hence, in this paper, we have reported the synthesis of polypyrrole nanoparticles and its characterization using FT-IR, UV-Vis spectrometer FESEM and their electrochemical properties was studied by cyclic voltammetry.

\section{Experimental}

Pyrrole, ammonium persulfate, methanol and sulphosalycylic acid were purchased from Qualigens and used without further purification. Doubly distilled water was used as the solvent.

\section{Synthesis of polypyrrole nanoparticles}

Reaction of pyrrole $(10 \mathrm{mM})$ with ammonium persulphate $(14 \mathrm{mM})$ yielded polypyrrole in doubly distilled water as solvent. The reaction mixture was allowed to stir for $8 \mathrm{~h}$ and under the ice cooling conditions. After that, the final product was filtered and washed with methanol and distilled water (50:50) to remove any unreacted pyrrole and ammonium persulphate.

\section{Characterization methods}

FT-IR spectrum of the polymer was recorded on Schimadzu FT-IR 8300 series instrument by using potassium bromide pellets. UV-Visible spectrum was recorded using a PerkinElmer 320 spectrophotometer from 200 to $800 \mathrm{~nm}$. The morphology of the polymer was analyzed by FE-SEM using a HITACHI SU6600 field emissionscanning electron microscopy. The electrochemical experiments were performed on a CHI 600A electrochemical instrument using the as-modified electrode and bare GCE as working electrode, a platinum wire was the counter electrode and saturated calomel electrode (SCE) was the reference electrode.

\section{Preparation of polypyrrole modified GCE}

Ultrasonically agitated solution of polypyrrole nanoparticles in water for $30 \mathrm{~min}$ was used for the coating. $5 \mu \mathrm{L}$ of the polypyrrole nanoparticles suspension was coated on the polished GCE and dried at room temperature.

\section{Results and Discussion}

\section{Structure and morphological characterization}

FT-IR spectrum of polypyrrole nanoparticles synthesized by chemical polymerization method is shown in the Figure 1. The Figure 1 shows the peaks at 1548 and $1466 \mathrm{~cm}^{-1}$ with respect to symmetric and antisymmetric aromatic ring stretching modes. The peaks at 1050 and $1300 \mathrm{~cm}^{-1}$ correspond to the $\mathrm{C}-\mathrm{H}$ and $\mathrm{C}-\mathrm{N}$ stretching vibrations respectively. The presence of two peaks at $1190 \mathrm{~cm}^{-1}$ was attributed to the $\mathrm{so}_{3}{ }^{-}$aromatic ring-doped state of polypyrrole. All of the IR peaks are in accordance with the expected structure for polypyrrole nanoparticles ${ }^{7,8}$.

The UV-Visible spectrum of polypyrrole nanoparticles is shown in Figure 2. The spectrum of polypyrrole nanoparticles shows two distinct bands at 381 and $572 \mathrm{~nm}$. These two transitions corresponded to the transitions from valence bond to polarons and bipolarons of the oxidized form of polypyrrole nanoparticles ${ }^{9}$. 


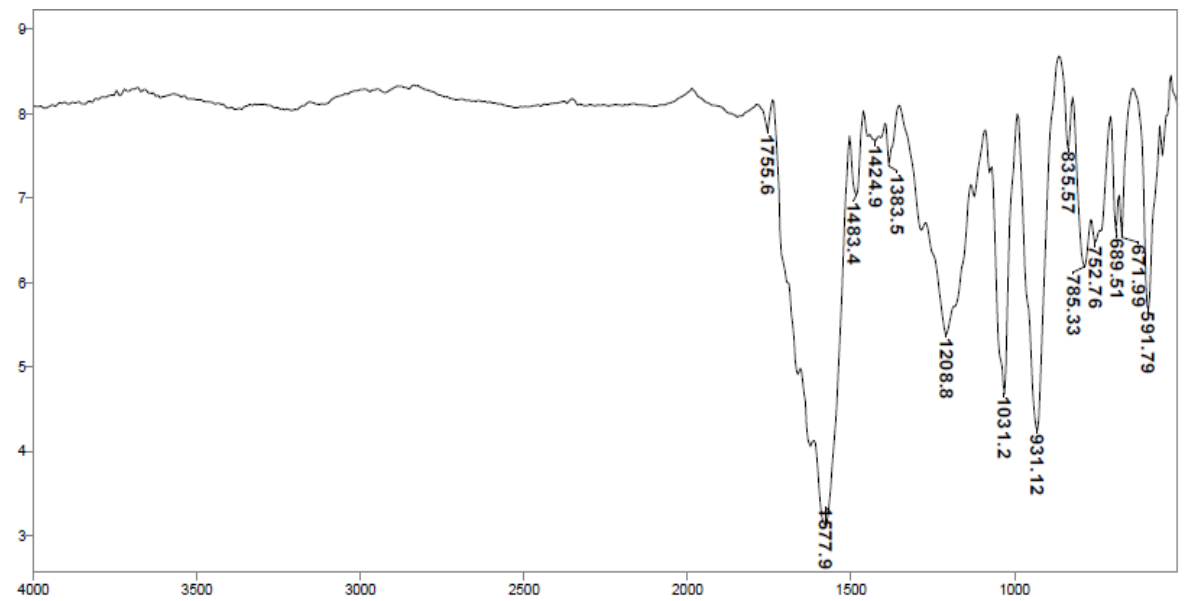

Figure 1. FT-IR spectrum of polypyrrole nanoparticles

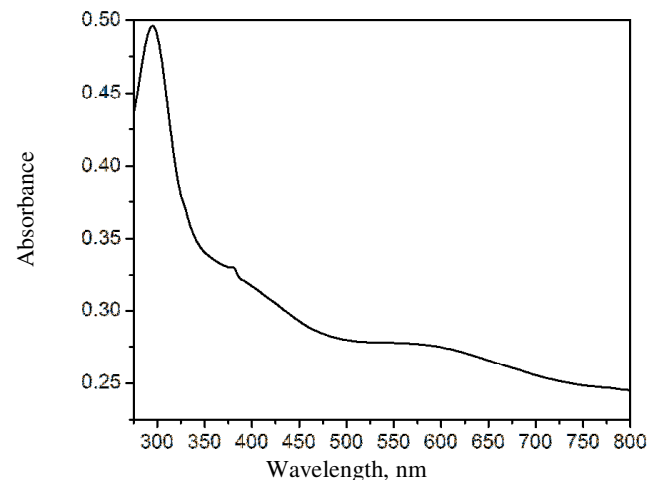

Figure 2. UV-Visible spectrum of polypyrrole nanoparticles

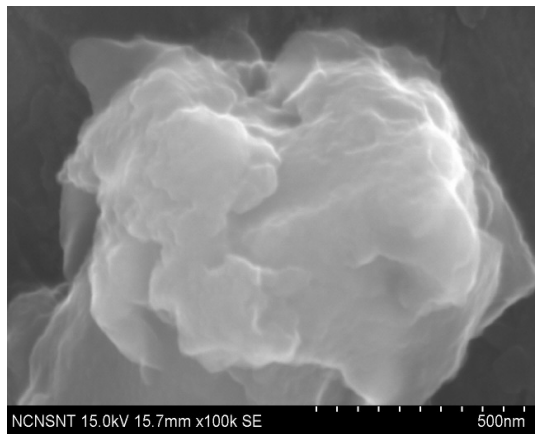

Figure 3. FESEM image of polypyrrole nanoparticles

The FESEM image of polypyrrole nanoparticles is shown in Figure 3. It shows that the synthesized polypyrrole is agglomerated by several nanoparticles.

Electroactivity is one of the most important properties of polypyrrole and is usually determined as a thin film coated on the surface of electrodes such as glassy carbon or platinum. The obtained two redox peaks is attributed to anion and cation movement respectively Figure 4 . The cyclic voltammograms $(\mathrm{CV})$ of polypyrrole revealed the normal oxidation/reduction processes according to:
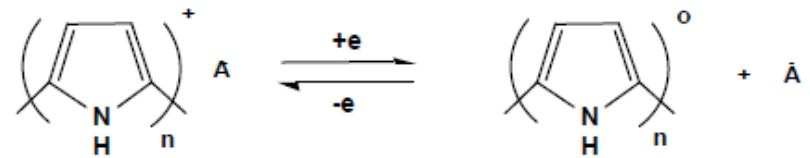

The auxiliary electrode reactions, during the redox reactions of polymer at the working electrode, will be the reduction of water or oxidation of water. The electrochemical reaction of polypyrrole involves the transport of ions in and out of the polymer in order to compensate the cationic nature of oxidised polymer ${ }^{10}$. Therefore, the redox reaction of polypyrrole involves both electron-transfer reaction and mass transport ${ }^{11}$. 


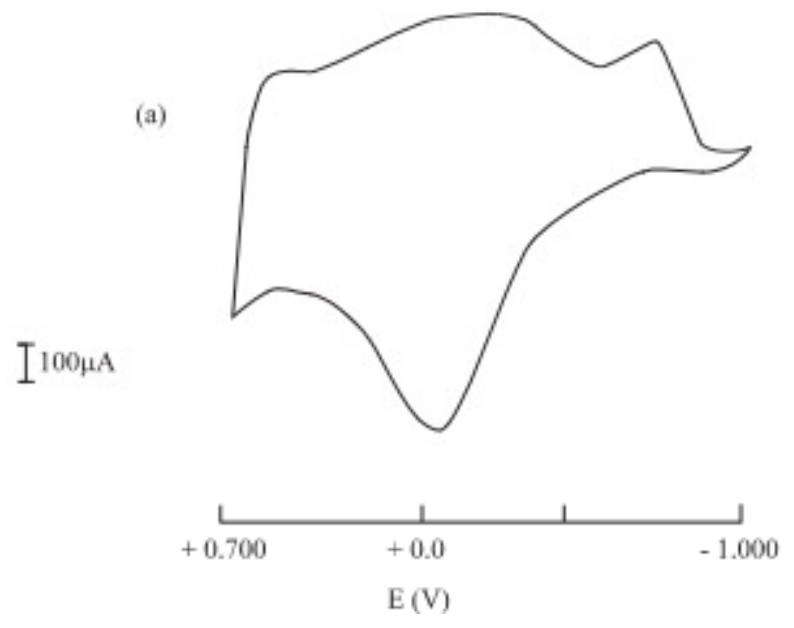

Figure 4. $\mathrm{CV}$ of polypyrrole nanoparticles in $0.1 \mathrm{M} \mathrm{NaCl}$ at $50 \mathrm{mV} / \mathrm{s}$

\section{Conclusion}

The polypyrrole were synthesized by rapid mixing method. The FT IR spectrum confirms the formation of polypyrrole. It also confirms that SSA is incorporated within the polypyrrole. UV-Visible spectra showed the characteristic peaks of presence of polypyrrole. Cyclic voltammogram shows that the polypyrrole nanoparticles have electrochemical activity. The modified electrode prepared will have potential application in quantitative determination of biomolecules.

\section{Acknowledgment}

One of the authors (SM) acknowledges the UGC-CPEPA for the financial assistance. We acknowledge the FE-SEM facility provided by the National Centre for Nanoscience and Nanotechnology, University of Madras.

\section{References}

1. Davidson K, Educ Chem., 1991, 28, 155.

2. Nishino A, J Power Sources, 1996, 60, 137-147.

3. Ferraris J P, Eissa M M, Brotherston I D, Loveday D C and Moxey A A, J Electroanal Chem., 1998, 459, 57-69.

4. Gordon M S, Chem Phys Lett., 1980, 76, 163-168.

5. Tourillon G and Garnier F, J Electrochem Soc., 1983, 130, 2042.

6. Ivory D M, Miller G G, Sowa J M, Shacklette L W, Chance R R and Baughman R H, J Chem Phys., 1979, 71, 1506.

7. Kharat H J, Kakade K P, Savale P A, Dutta K, Ghosh P and Shirsat M D, Polym Adv Technol., 2007, 18(5), 397-402.

8. Tian B and Zerbi G, J Chem Phy., 2009, 92(6), 3886-3891.

9. Wang Z L, Kong X Y, Ding Y, Gao P, Hughes W L, Yang R and Zhang Y, Adv Funct Mater., 2004, 14, 943-956.

10. Ansari R, Russian J Electrochem., 2005, 41(9), 1071-1078.

11. Ansari Khalkhali R, Price W E and Wallace G G, React Funct Polym., 2003, 56, 141-146. 